\title{
REPRESENTAÇÕES SOCIAIS DE ENFERMEIROS DA ATENÇÃO PRIMÁRIA À SAÚDE SOBRE CUIDADO DE ENFERMAGEM NO PÓS-PARTO*
}

\author{
Sibele Lima da Costa Dantas", Dafne Paiva Rodrigues², Ana Virgínia de Melo Fialho², Eryjosy \\ Marculino Guerreiro Barbosa3, Ana Maria Martins Pereira1', Nayara Sousa de Mesquita
}

\begin{abstract}
RESUMO: Objetivo: apreender as representações sociais de enfermeiros da Atenção Primária à Saúde sobre o cuidado de enfermagem no pós-parto. Método: estudo qualitativo, norteado pela Teoria das Representações Sociais, desenvolvido de setembro a outubro de 2015, com 31 enfermeiros da Estratégia Saúde da Família do município de Mossoró, Rio Grande do Norte, Brasil, por meio de entrevista semiestruturada. Os dados foram analisados com o auxílio do software ALCESTE, que realiza análise lexical de dados textuais. Resultados: verificou-se que os enfermeiros ancoram o cuidado de enfermagem no puerpério como cuidados desenvolvidos ao recém-nascido, priorizando a consulta de puericultura em detrimento da puerperal. Conclusão: é necessário que os enfermeiros desenvolvam o cuidado de enfermagem no pós-parto de forma integral, direcionado para além dos procedimentos técnicos, por meio de escuta qualificada e atenÇão às necessidades biopsicossociais da puérpera.
\end{abstract}

DESCRITORES: Saúde da mulher; Período pós-parto; Cuidados de enfermagem; Estratégia Saúde da Família; Psicologia social.

\section{SOCIAL REPRESENTATIONS OF PRIMARY HEALTH CARE NURSES ABOUT NURSING CARE IN THE POSTPARTUM PERIOD}

ABSTRACT: Objective: to gain insight on the social representations of primary health care nurses about nursing care in the postpartum period. Method: Qualitative study based on the Theory of Social Representations, conducted from September to October 2015, with 31 nurses of the Family Health Strategy of the city of Mossoró, Rio Grande do Norte, Brazil, through semi-structured interviews. Data were analyzed with the aid of ALCESTE software, which conducts lexical analysis of text data. Results: It was found that the care provided by nurses prioritizes childcare appointments (care to the newborn) to the detriment of postpartum appointments. Conclusion: Nurses should develop integral postpartum care, which goes beyond the implementation of technical procedures, involving qualified listening and care to the biopsychosocial needs of postpartum women.

KEYWORDS: Women's health; Postpartum period; Nursing care; Family Health Strategy; Social Psychology

\section{REPRESENTACIONES SOCIALES DE ENFERMEROS DE ATENCIÓN PRIMARIA DE SALUD SOBRE ATENCIÓN DE ENFERMERÍA EN POSPARTO}

RESUMEN: Objetivo: Comprender las representaciones sociales de enfermero de Atención Primaria de Salud sobre la atención de enfermería en el posparto. Método: Estudio cualitativo, orientado por la Teoría de las Representaciones Sociales, desarrollado de setiembre a octubre de 2015 con 31 enfermeros de la Estrategia Salud de la Familia del municipio de Mossoró, Rio Grande do Norte, Brasil, aplicándose entrevista semiestructurada. Datos analizados con ayuda del software ALCESTE, que efectúa un análisis lexical de los datos textuales. Resultados: Se verificó que los enfermeros enfocan la atención de enfermería durante el puerperio como cuidados brindados al recién nacido, priorizando la consulta de puericultura por sobre la puerperal. Conclusión: Es necesario que los enfermeros efectúen la atención de enfermería durante el posparto de manera integral, orientado más allá de los procedimientos técnicos, a través de una escucha calificada y atención de las necesidades biopsicosociales de la puérpera.

DESCRIPTORES: Salud de la Mujer; Periodo Posparto; Atención de Enfermería; Estrategia de Salud Familiar; Psicología Social.

\footnotetext{
*Artigo extraído da dissertação de mestrado intitulada: Cuidado de Enfermagem no Período Pós-Parto: Representações Sociais de Enfermeiros e Puérperas na Atenção Primária à Saúde. Universidade Estadual do Ceará, 2016.

${ }^{1}$ Enfermeira. Doutoranda em Cuidados Clínicos em Enfermagem e Saúde. Universidade Estadual do Ceará. Fortaleza, CE, Brasil. ${ }^{2}$ Enfermeira. Doutora em Enfermagem, Docente do Programa de Pós-Graduação Cuidados Clínicos em Enfermagem e Saúde. Universidade Estadual do Ceará. Fortaleza, CE, Brasil.

${ }^{3}$ Enfermeira. Doutora em Cuidados Clínicos em Enfermagem e Saúde. Docente do Departamento de Enfermagem no Centro Universitário Unichristus e na Faculdade Integrada Grande Fortaleza. Fortaleza, CE, Brasil.
}

Av. Dr. Silas Munguba, 1700 - Campus do Itaperi, CEP 60741-000 - Fortaleza, Ceará, Brasil.

sibelelcosta@gmail.com 


\section{INTRODUÇÃO}

O puerpério compreende a fase do ciclo gravídico-puerperal que tem início logo após o parto e a expulsão da placenta até a volta do organismo às condições pré-gravídicas, passível de involução, com duração média de 6 a 8 semanas. Considerada uma fase de novos aprendizados, de consolidação da unidade familiar e de laços afetivos, o puerpério pode também apresentar riscos e requer assistência qualificada, com a finalidade de melhoria das condições de saúde, evitando, assim, complicações obstétricas $^{(1-2)}$.

Apesar das evidentes demandas de cuidados durante esse período, sabe-se que, universalmente, a atenção ao pré-natal e parto tem recebido maior investimento público do que a atenção puerperal. Os resultados de saúde após o parto, nos países desenvolvidos ou em desenvolvimento, apontam que há falta de reconhecimento sistemático de que o cuidado durante o puerpério é continuidade essencial do cuidado na gravidez e parto ${ }^{(3)}$.

A Organização Mundial de Saúde (OMS) idealiza que todas as mulheres recebam cuidados de qualidade durante toda a gravidez, parto e período pós-natal ${ }^{(4)}$, e orienta que os sistemas de saúde invistam na atenção primária à saúde (APS) como modalidade assistencial potencialmente capaz de melhorar os indicadores de saúde da população ${ }^{(5)}$.

Nesta perspectiva, e visando reorientar o modelo assistencial brasileiro, o Ministério da Saúde (MS) implantou a Estratégia de Saúde da Família (ESF), que iniciou com o programa Saúde da Família (PSF) em 1994, e, desde então, é definido como estratégia prioritária para a organização da APS no país ${ }^{(6)}$. A proposta da estratégia é fortalecer as ações de prevenção de agravos e doenças, reabilitar e promover a saúde da população, incluindo a saúde das mulheres durante o ciclo gravídico-puerperal, e, nessa direção, disponibilizar um serviço que esteja apto a atender às demandas inerentes a esse período.

Nesse contexto de aprendizagem e adaptações, é fundamental o cuidado clínico de enfermagem, pois a mulher se depara com os novos papéis assumidos diante da maternidade e precisa do suporte adequado a fim de superar as dificuldades encontradas e para que esse período transcorra sem complicações.

Depois da alta do bebê, uma visita domiciliar deve ser realizada logo na primeira semana, e uma consulta de controle pós-parto em até 42 dias após o final da gestação. O retorno da mulher e do recémnascido ao serviço de saúde deve ser estimulado desde o pré-natal, na maternidade e pelos agentes comunitários de saúde na visita domiciliar ${ }^{(7)}$.

A consulta de enfermagem no puerpério é um momento importante para a mulher esclarecer as suas dúvidas, aprender sobre o cuidado de si e do bebê, é uma oportunidade rica e propícia para os momentos de educação em saúde entre enfermeiro e puérpera, contribuindo para a promoção da saúde da mulher, do recém-nascido e da sua família ${ }^{(8)}$.

Diante desse contexto, surgiu a seguinte indagação: Quais as representações sociais dos enfermeiros da Estratégia Saúde da Família acerca do cuidado de enfermagem no pós-parto?

Verificada a necessidade de conceber como os profissionais que prestam assistência de enfermagem à puérpera entendem e se relacionam com essa realidade, recorreu-se à Teoria das Representações Sociais como aporte teórico para compreender e caracterizar o cuidado de enfermagem no pós-parto, a partir dos significados, conhecimentos e percepções construídas socialmente por enfermeiros acerca dessa assistência e, assim, inferir considerações relevantes sobre o objeto de estudo.

À medida que as representações sociais aproximam o universo do pesquisador do universo de significados construídos nas relações sociais dos sujeitos estudados, explicita-se a necessidade de trabalhar nas pesquisas em saúde com essa abordagem teórica, na perspectiva de desvelar um determinado fenômeno ${ }^{(9)}$.

Desse modo, o presente estudo objetivou apreender as representações sociais dos enfermeiros da Atenção Primária à Saúde sobre o cuidado de enfermagem no pós-parto. 


\section{MÉTODO}

Trata-se de pesquisa qualitativa, norteada pelo referencial da Teoria das Representações Sociais (TRS) de Serge Moscovici(10), realizada a partir dos dados coletados, nos meses de setembro e outubro de 2015, de 31 enfermeiros da Estratégia Saúde da Família do município de Mossoró, Rio Grande do Norte, Brasil.

Foram selecionados enfermeiros que realizavam assistência à saúde da mulher e estavam havia mais de 6 meses na Unidade de Atenção Primária à Saúde (UAPS), por entender que é um período que o profissional já realizou o reconhecimento do território, desenvolvendo vínculo com a população. Foram excluídos os que estavam em gozo de férias ou licença-maternidade.

As entrevistas aconteceram individualmente, na própria unidade de saúde, em horário de trabalho. Utilizou-se de um instrumento para traçar o perfil social, profissional e acadêmico dos enfermeiros participantes, seguido de um roteiro de entrevista semiestruturado com as seguintes questões norteadoras: 1. O que é para você o cuidado de enfermagem no puerpério? 2. Como ocorre esse acompanhamento? 3. O que você pensa que é para os outros o cuidado de enfermagem no puerpério?

Os dados referentes ao perfil dos participantes foram digitados em uma planilha do Microsoft Excel, versão 2010, e analisados por meio de estatística descritiva simples, apresentando, assim, as características da população estudada. O material proveniente da entrevista semiestruturada foi submetido à análise lexical, utilizando o software Alceste(Analyse Lexicale par Contexte d'un Ensemble de Segments de Texte), versão 2012, o qual emprega uma análise de Classificação Hierárquica Descendente e possibilita uma análise lexicográfica do material textual, oferecendo contextos (classes lexicais), que são caracterizados pelo seu vocabulário e pelos segmentos de texto que compartilham esse vocabulário ${ }^{(11-13)}$.

Cada entrevista foi considerada uma unidade de contexto inicial $(\mathrm{UCI})$, e, portanto, o conjunto de $\mathrm{UCI}$ constituiu o corpus de análise processado pelo programa. O Alceste dividiu o material em Unidades de Contexto Elementar (UCE), que são pequenos segmentos de entrevista, e, de acordo com a distribuição do vocabulário nessas unidades é que se formaram as classes de palavras.

Assim, cada classe de UCE apresenta vocabulário semelhante entre si e vocabulário diferente das UCE de outras classes. A significância estatística dos vocábulos nas classes é representada pelo valor de "Phi", indicando que quanto maior o valor, mais relevante é a palavra na classe construída.

A fim de preservar o anonimato dos participantes da pesquisa, as falas foram identificadas com a letra "E", seguida da sequência cronológica das entrevistas realizadas. Este estudo foi pautado pelos parâmetros da Resolução 466/12 $2^{(14)}$, do Conselho Nacional de Saúde do Ministério da Saúde, que dispõe sobre a pesquisa com seres humanos, foi submetido à avaliação do Comitê de Ética em Pesquisa da Universidade Potiguar (CEP/UnP), e só teve início após a aprovação do projeto sob o parecer $\mathrm{n} . .^{\circ}$ 1.054.847 de 06/05/2015 e a autorização da Secretaria Municipal de Saúde.

\section{RESUltADOS}

No tocante ao perfil social, profissional e acadêmico dos 31 enfermeiros que participaram da entrevista, $27(87,1 \%)$ eram do sexo feminino, $13(41,9 \%)$ estavam na faixa etária entre 36 e 44 anos, $21(67,7 \%)$ eram casados, $23(74,2 \%)$ possuíam filho, $13(41,9 \%)$ apresentavam de 6 a 10 anos de experiência em UAPS, 17 (54,8\%) possuíam outro vínculo empregatício e 29 (93,5\%) tinham titulação de especialista, mas apenas 13 (41,9\%) possuíam especialização na área de Saúde da Família.

A análise do corpus pelo Alceste obteve $31 \mathrm{UCl}$ e, a partir das 251 palavras analisáveis e 267 UCE classificadas (representando $71 \%$ de aproveitamento do material), geraram-se quatro classes, apresentadas no Dendograma de Classes Hierarquicamente Descendentes (CHD, Figura 1). 


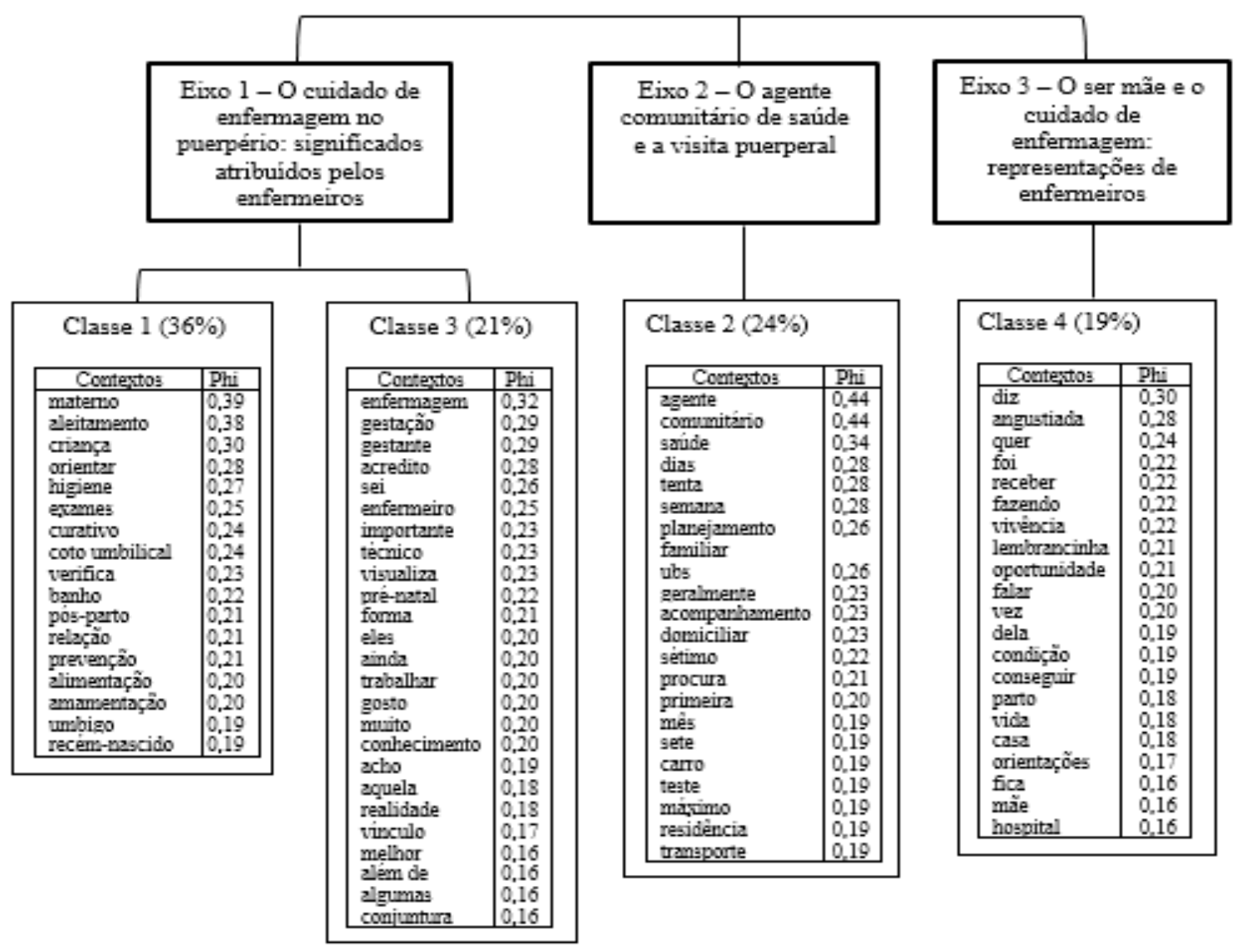

Figura 1 - Representações sociais do cuidado de enfermagem no pós-parto. Mossoró, RN, Brasil, 2015

A classe 1 (36\% das UCE) é composta das palavras materno, aleitamento, criança, higiene, curativo e coto umbilical, e está associada à classe 3 (21\% das UCE), formada por enfermagem, gestação, enfermeiro, importante, pré-natal, conhecimento e vínculo. A classe 2 (24\% das UCE) tem como palavras mais ilustrativas: agente, comunitário, saúde, acompanhamento, domiciliar, sétimo e transporte. Já a classe 4 (19\% das UCE) é formada por angustiada, vivência, lembrancinha, oportunidade e orientações.

Os trechos das UCE características da classe 1 descrevem como o enfermeiro vê o cuidado de enfermagem no puerpério, destacando-se os cuidados com o recém-nascido:

É a assistência total, o primeiro cuidado com o recém-nascido, ver como é que ele está. Vendo ele como um todo, o corpo, a cicatriz umbilical, se já ocorreu a queda do umbigo, se está mamando, como está mamando, se está fazendo a pega normal. (E25)

A gente trabalha no puerpério mais com a criança, com o recém-nascido [...]. (E30)

As UCE características da classe 3 demonstram que, para o enfermeiro, a continuidade do cuidado de enfermagem no puerpério é resultante do vínculo estabelecido entre o enfermeiro e a mulher durante as consultas do pré-natal:

Se você acompanha uma gestante todo o pré-natal, nove consultas, seis consultas, nesse período todo você já conhece bastante ela para, no puerpério, se vir alguma alteração, alguma coisa que possa provocar alguma patologia [...]. (E17)

Por isso, é importante a continuidade do cuidado iniciado no pré-natal, quando o enfermeiro conhece a realidade dessa mulher, até porque aproximação é diferente de vínculo, nós estabelecemos vínculo com a puérpera. (E31)

Ao analisar as UCE e o contexto semântico dessa classe, ficou evidente que os enfermeiros compreendem a essencialidade do trabalho em equipe para garantir assistência integral e humanizada às puérperas. Porém, ao comentar a dinâmica de trabalho na equipe de saúde, demonstraram certo 
desânimo, uma vez que há dificuldades em realizar ações em conjunto com os demais profissionais, além daqueles que compõem a equipe de enfermagem, tornando o cuidado fragmentado:

Esse cuidado de enfermagem é de extrema relevância, uma vez que a enfermagem está mais perto dessa mulher, lidando diariamente com essa mulher, não só o enfermeiro, a equipe de enfermagem. (E8)

Esse cuidado está fragmentado, principalmente, porque a utopia de equipe seria uma equipe multiprofissional, com olhares diferenciados para aquela puérpera, essa equipe não existe. Então o cuidado de enfermagem, ele se fragmenta de certa forma. (E8)

Os trechos das UCE características da classe 2 expressam que os enfermeiros percebem o puerpério como um período crítico e que reconhecem a necessidade da visita logo na primeira semana após o parto, porém, diante da indisponibilidade de transporte na equipe de saúde, essa visita é realizada pelo agente comunitário de saúde (ACS), que segue a recomendação de orientar a puérpera a procurar a unidade de saúde para realizar a consulta puerperal:

Quando eu não consigo fazer a visita, eu falo com a agente comunitária de saúde para encaminhar essa mulher, e ela vem a até a Unidade Básica de Saúde [...] (E16)

Foi evidenciado, ainda, que, quando não é possível realizar a visita ou o agendamento da consulta -, pois algumas puérperas vão para a residência de parentes ao sair da maternidade -, os enfermeiros aproveitam os momentos oportunos em que a puérpera comparece à UAPS para realizar algum procedimento para dialogar com ela sobre o pós-parto.

A gente faz as visitas domiciliares, e quando não dá para fazer a visita, a gente pega ela no dia que vem fazer o teste do pezinho, porque a gente faz o teste quarta-feira pela manhã [...] (E20)

As UCE características da classe 4 descrevem que as enfermeiras que tiveram a oportunidade de realizar atendimento à mulher, tanto na atenção primária quanto na maternidade, demonstraram compreender a puérpera em um contexto mais amplo do que aquele visualizado durante a consulta puerperal:

Então, você espera que a mãe esteja lá toda feliz, cuidando do bebê, amamentando exclusivamente, cumprindo todas aquelas orientações que a gente fez no pré-natal e quando a gente vê a condição do parto, o que ela passou naquele ambiente hospitalar [...] (E4)

[...] quando a mulher tem uma experiência negativa anterior, com dificuldade, a gente tem que intervir, então, por isso que é importante ver cada uma, ver cada realidade. (E1)

Outro aspecto relevante nessa classe foi o cuidado de enfermagem influenciado pela experiência pessoal da enfermeira que já passou pelas adaptações da maternidade:

[...] então, às vezes eu olho pra uma mãe que diz que não tem leite, e eu entendo, porque eu também não tive leite, tomei tudo o que você imaginar, que os médicos mandaram tomar, pesquisei, mas eu não tive leite, então meu puerpério também não foi bom, eu tive início de depressão pós-parto. (E13)

\section{DISCUSSÃO}

Ao analisar os conteúdos lexicais mencionados pelos enfermeiros, bem como as palavras que obtiveram maior associação com a classe 1, percebe-se que o cuidado de enfermagem no puerpério está ancorado nos cuidados ao recém-nascido. Há uma predominância de conteúdos relacionados à consulta de puericultura sobrepondo a consulta puerperal, o que pode gerar, ocasionalmente, negligência no que se refere à atenção às necessidades da puérpera, provenientes dessa fase do ciclo gravídico-puerperal. Até mesmo a intensificação dos cuidados relacionados às mamas, no sentido de garantir amamentação eficaz, reflete a preocupação prioritária com o desenvolvimento saudável do recém-nascido.

O contato contínuo entre mãe e filho por meio da amamentação fortalece os laços afetivos, o envolvimento dos familiares e o prolongamento dessa prática. A amamentação bem-sucedida desperta na mulher um sentimento de ligação profunda com o filho e de realização como mulher e mãe, e, se 
em algum momento essa ligação é desfeita, seja por qualquer motivo, esse momento pode ter outros $\operatorname{sentidos}^{(15)}$. Faz-se necessário que o profissional de saúde realize os cuidados à puérpera para além dos aspectos fisiológicos da mama e da amamentação em si, buscando conhecer os significados do ato de amamentar para a puérpera e qual a representação construída sobre amamentação, levando em consideração os aspectos pessoais, sociais e culturais.

Ainda no mesmo eixo 1, "O cuidado de enfermagem no puerpério: significados atribuídos pelos enfermeiros", há a classe 3, em que os conteúdos apresentados podem justificar o motivo pelo qual o recém-nascido tem sido o foco principal do cuidado de enfermagem no período pós-parto. Os enfermeiros mencionam que ao acompanhar as mulheres desde o pré-natal é mais fácil identificar alguma alteração que possa ocorrer durante o puerpério, ressaltando a importância do vínculo estabelecido entre profissional e usuária. Dessa forma, o recém-nascido seria "algo" não familiar. É nesse contexto que a ancoragem, um dos processos que geram representações sociais, é concebida, com a finalidade de transformar algo estranho em familiar, comum $^{(10)}$.

A mesma classe evidenciou que os enfermeiros entendem que, apesar de organizacionalmente complexo, é o trabalho em equipe que garantirá à mulher o cuidado integral no puerpério. É necessário, portanto, que os cuidados de enfermagem durante o período pós-parto sejam direcionados sempre ao binômio mãe-bebê, inclusive, interrogando à mãe se ela tem alguma dúvida ou queixa, dando seguimento às demais etapas da consulta, proporcionando cuidado integral.

Considerando que a integralidade é um dos princípios doutrinários do Sistema Único de Saúde (SUS), o trabalho em equipe representa um dos principais pilares para a efetivação do cuidado integral nos serviços de saúde. Assim, uma abordagem integral do binômio mãe-filho, no período pós-parto pode ser facilitada pela soma de olhares dos distintos profissionais que compõem a equipe de Saúde da Família, favorecendo uma ação interdisciplinar ${ }^{(16)}$.

A ação interdisciplinar está ancorada na possibilidade da prática de um profissional se reconstruir na prática do outro, ambos sendo transformados para a intervenção na realidade em que estão inseridos, contribuindo para uma abordagem ampla e resolutiva do cuidado ${ }^{(17)}$.

Nos contextos lexicais e palavras analisáveis da classe 2, que pertence ao eixo 2, "O agente comunitário de saúde e a visita puerperal", observou-se que, apesar de os enfermeiros descreverem a importância da visita domiciliar à puérpera logo na primeira semana após o parto, diante da conjuntura adversa, no que se refere à dificuldade de transporte nas equipes de saúde, a visita domiciliar no puerpério tem sido realizada somente pelo ACS. Percebeu-se também que, dessa forma, o retorno da mulher e do recémnascido à unidade de saúde fica fragilizado.

A maioria das complicações maternas e neonatais acontece na primeira semana após o parto, portanto, o retorno da mulher e do recém-nascido ao serviço de saúde deve acontecer logo nesse período. Os enfermeiros devem estar atentos e preparados para aproveitar a oportunidade de contato com a mulher e o recém-nascido nesse período para instituir todo cuidado previsto ${ }^{(18)}$. Quando a mulher e o recém-nascido retornam à unidade de saúde, é possível reavaliar as suas condições de saúde, o registro das alterações e da amamentação, o retorno da menstruação e da atividade sexual, realizar ações educativas, enfim, investigar e conduzir possíveis intercorrências ${ }^{(7)}$.

É importante que a consulta de enfermagem no pós-parto seja feita de forma sistemática, pois essa, além de possibilitar o seguimento clínico-educativo da mulher e do recém-nascido, também propicia o acolhimento que favorece a vinculação da mulher, do pai e da família ao serviço e a abordagem de suas necessidades ${ }^{(19)}$.

A classe 4, que compõe o eixo 3 "O ser mãe e o cuidado de enfermagem: representações de enfermeiros", apresenta uma ressignificação do cuidado de enfermagem no puerpério quando as enfermeiras relatam ter acompanhado a mulher durante o pré-natal e no momento do parto, por terem vínculos na atenção primária e no âmbito hospitalar. Outra reconstrução das representações sociais ocorre na perspectiva da enfermeira que ancora o cuidado de enfermagem no puerpério a partir das suas vivências pessoais, do seu universo consensual.

O universo consensual, também chamado de senso comum, constrói as representações sociais a partir das contradições sociais diárias e se inter-relaciona com o universo reificado, caracterizado pelo conhecimento científico, objetividade e rigor metodológico, dando forma à nossa realidade ${ }^{(10)}$. 
Na fase do puerpério, a mulher sente necessidade de ser apoiada em seus medos e inseguranças, é possível que ela apresente limitações emocionais e físicas que dificultem o cuidado ao seu filho ${ }^{(20)}$. O momento do retorno é quando muitas mulheres se sentem angustiadas, desamparadas, e por vezes despreparadas para assumir a maternidade. Portanto, é de fundamental importância que o cuidado de enfermagem nesse período esteja direcionado às necessidades da puérpera em todos os aspectos.

O estudo realizado apresentou limitações quanto à restrita abrangência demográfica e ao número reduzido da amostra, não permitindo uma extrapolação dos resultados. Sugere-se, portanto, futuras investigações utilizando amostras mais amplas e abrangendo outras regiões demográficas.

\section{CONCLUSÃO}

O cuidado de enfermagem no pós-parto foi representado, pelos enfermeiros neste estudo, como aquele que é direcionado, prioritariamente, aos cuidados com o recém-nascido. À puérpera são dadas orientações gerais para o desenvolvimento da maternidade, evidenciando-se o foco na amamentação.

O agente comunitário de saúde exerce papel importante para comunicar o retorno da puérpera à sua residência, após a alta da maternidade, para que seja dado seguimento ao cuidado dispensado à mulher nesse período.

Evidenciou-se, também, que a experiência pessoal da enfermeira, enquanto puérpera gerou uma ressignificação social da importância do cuidado de enfermagem desenvolvido no pós-parto. Os conhecimentos obtidos no universo reificado passam ao mundo consensual, adaptando-se aos usos específicos deste mundo prático.

É necessário que os enfermeiros possam desenvolver o cuidado de enfermagem no pós-parto de forma integral, direcionando o cuidado para além dos procedimentos técnicos, buscando utilizar-se da escuta qualificada como ferramenta de cuidado e atentando para as necessidades biopsicossociais da puérpera.

O estudo servirá, portanto, como um dispositivo importante a ser utilizado pelos enfermeiros da Atenção Primária à Saúde, gerando novos conhecimentos e um novo olhar no tocante ao atendimento à mulher dentro do ciclo reprodutivo, mais especificamente no período pós-parto. Espera-se que a partir dos resultados obtidos com este trabalho sejam fomentadas discussões acerca do cuidado de enfermagem no pós-parto, proporcionando mudanças positivas na prática do enfermeiro e, consequentemente, garantindo às puérperas um atendimento de qualidade e que corresponda às suas necessidades de cuidado.

\section{REFERÊNCIAS}

1. Mazzo MHSN, de Brito RS, de Souza NL, Gama AP. Taking care of the puerpera after hospital discharge: a literature review. J. Nurs. UFPE on line. [Internet] 2012;6(11) [acesso em 17 mar 2017]. Disponível: http://dx.doi.org/10.5205/ reuol.2185-16342-1-LE.0611201227.

2. Garcia ESGF, Leite EPRCL, Nogueira DAN. Nursing care to puerperal women in primary care units. J. Nurs. UFPE online. [Internet] 2013;7(10) [acesso em 17 mar 2017]. Disponível: https://periodicos.ufpe.br/revistas/revistaenfermagem/ article/view/12218.

3. Osava RH. A continuidade do cuidado a mulher e recém-nascido após o parto e nascimento: perspectivas de um cuidado integrador e autonomizante. In: VII Congresso Brasileiro de Enfermagem Obstétrica e Neonatal. I Congresso Internacional de Enfermagem Obstétrica e Neonatal; Minas Gerais, Brasil. Minas Gerais: ABENFO-MG; 2011.

4. Organização Mundial da Saúde (OMS). Recomendações da OMS sobre cuidados pré-natais para uma experiência positiva na gravidez. [Internet] Geneva: OMS; 2016 [acesso em 01 ago2017]. Disponível em: http://apps.who.int/iris/ bitstream/10665/250800/2/WHO-RHR-16.12-por.pdf.

5. Ferreira JBB, Borges MJG, dos Santos LLD, Forster AC. Internações por condições sensíveis à atenção primária à saúde em uma região de saúde paulista, 2008 a 2010. Epidemiol. Serv. Saúde [Internet] 2014;23(1) [acesso 01 ago 2017]. Disponível: http://dx.doi.org/10.5123/S1679-49742014000100005. 
6. Ministério da Saúde (BR). Secretaria de Atenção à Saúde. Departamento de Atenção Básica. O trabalho do agente comunitário de saúde. [Internet] Brasília: Ministério da Saúde; 2009 [acesso em 01 ago 2017]. Disponível: http://189.28.128.100/dab/docs/publicacoes/geral/manual_acs.pdf.

7. Ministério da Saúde (BR). Secretaria de Atenção à Saúde. Departamento de Atenção Básica. Atenção ao pré-natal de baixo risco. [Internet] Brasília: Ministério da Saúde, 2013 [acesso em 01 ago 2017]. Disponível: http://bvsms.saude.gov. br/bvs/publicacoes/cadernos_atencao_basica_32_prenatal.pdf.

8. Ribeiro DHF, Lunardi VL, Gomes GC, Xavier DM, Chagas MCDS. Experiences of woman's care: the report of puerperal women. J. Nurs. UFPE online. [Internet] 2014;8(4) [acesso em 17 mar 2017]. Disponível: https://periodicos. ufpe.br/revistas/revistaenfermagem/article/view/9748.

9. Bittencourt IS, Vilela ABA. Representações sociais: uma abordagem teórica em saúde. Rev. bras. promoç. saúde. [Internet] 2011;24(1). [acesso em 17 mar 2017]. Disponível: http://www.redalyc.org/articulo.oa?id=40819112012.

10. Moscovici S. Representações sociais: investigações em psicologia social. 9ạ ed. Petrópolis: Vozes, 2012. O fenômeno das representações sociais. p.29-109.

11. Saraiva ERA, Coutinho MPL, Miranda RS. O emprego do software Alceste e o desvendar do mundo lexical em pesquisa documental. In: Coutinho MPL, Saraiva ERA. Métodos de pesquisa em psicologia social: perspectivas qualitativas e quantitativas. João Pessoa: UFPB; 2011. p.95-106.

12. Camargo BV. Alceste: um programa informático de análise quantitativa de dados textuais. In: Moreira ASP et al. (org.). Perspectivas teórico-metodológicas em representações sociais. João Pessoa: Editora Universitária UFPB, 2005. p.511-539.

13. Reinert M. Alceste une methodologie d'analyse des donnes textuelles et uneapplication: Aurelia de Gerard de Nerval. Bull Methodol. Sociol. [Internet] 1990;(26) [acesso em 01 ago 2017]. Disponível : https://doi-org.ez76.periodicos.capes. gov.br/10.1177/075910639002600103.

14. Ministério da Saúde (BR). Conselho Nacional de Saúde. Diretrizes e normas regulamentadoras de pesquisa envolvendo seres humanos. Resolução n. 466, de 12 de dezembro de 2012. Brasília; 2012.

15. Dias RB, Boery RNSO, Vilela ABA. Conhecimento de enfermeiras e estratégias de incentivo da participação familiar na amamentação. Ciênc. saúde coletiva. [Internet] 2016;21(8) [acesso em 15 jun 2017]. Disponível: http://dx.doi. org/10.1590/1413-81232015218.08942015.

16. Viegas SMF, Penna CMM. A construção da integralidade no trabalho cotidiano da equipe saúde da família. Esc. Anna Nery. [Internet] 2013;17(1) [acesso em 15 jun 2017]. Disponível: http://dx.doi.org/10.1590/S1414-81452013000100019.

17. Araújo MBS, Rocha PM. Trabalho em equipe: um desafio para a consolidação da estratégia de saúde da família. Ciênc. saúde coletiva. [Internet] 2007;12(2) [acesso em 15 jun 2017]. Disponível: http://dx.doi.org/10.1590/S141381232007000200022 .

18. São Paulo (Estado). Secretaria da Saúde. Coordenadoria de planejamento em saúde. Assessoria técnica em saúde da mulher. Atenção à gestante e puérpera no SUS-SP: manual técnico do pré-natal e puerpério. [Internet] São Paulo: SES/SP; 2010 [acesso em 26 mai 2018]. Disponível: http://www.saude.sp.gov.br/resources/ses/perfil/gestor/destaques/ atencao-a-gestante-e-a-puerpera-no-sus-sp/manual-tecnico-do-pre-natal-e-puerperio/manual_tecnicoii.pdf.

19. Oliveira DC, Mandu ENT, Corrêa ACP, Tomiyoshi JT, TeixeiraRC. Estrutura organizacional da atenção pós-parto na estratégia saúde da família. Esc. Anna Nery. [Internet] 2013;17(3) [acesso em 03 abr 2017]. Disponível: http://dx.doi. org/10.1590/S1414-81452013000300007.

20. Souza CD, Monteiro LF, Rodrigues SBMA, Munhoz GMA, Lisboa SR. Vivencias y significados del primer baño dado por la puérpera a su hijo recién nacido. Enferm. glob. [Internet] 2010;(19) [acesso em 03 abr 2017]. Disponível: http:// scielo.isciii.es/pdf/eg/n19/pt_docencia3.pdf. 